\title{
AVANCES DEL DISEÑO DE UNA ESTRATEGIA DIDÁCTICA PARA LA APLICACIÓN DEL MODELO HOLÍSTICO DE LIDERAZGO POLICIAL - MHLP, DESDE LA PRÁCTICA DOCENTE EN LA ESCUELA DE CARABINEROS PROVINCIA DE VÉLEZ
}

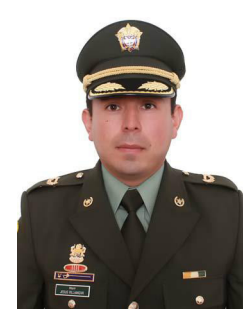

\author{
Jesús Enrique Villamizar Suárez ${ }^{1}$ \\ Policía Nacional de Colombia \\ jesus.villamizar@correo.policia.gov.co
}

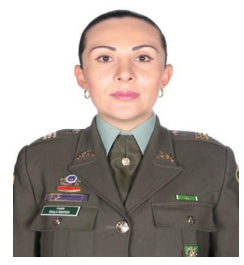
Sandra Paola Riveros Guzmán²
Policía Nacional de Colombia
Sandra.riveros@correo.policia.gov.co

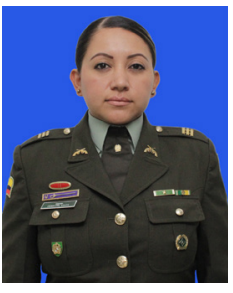

\section{Diomayra Lorena Vacca Guerrero ${ }^{3}$ \\ Policía Nacional de Colombia \\ diomayra.vacca1016@correo.policia.gov.co}

\section{RESUMEN}

Este artículo es un avance de la investigación relacionada con la aplicación del Modelo Holístico de Liderazgo Policial (MHLP) de la Policía Nacional de Colombia (PONAL), en la Escuela de Carabineros Provincia de Vélez (ESVEL). En el caso de las escuelas de formación policial, aún no se han desarrollado procesos del MHLP. Por ello, ante esta necesidad y como un aporte a los docentes de la Escuela de Carabineros Provincia de Vélez, se está desarrollando el trabajo de investigación que antecede el presente artículo, cuya pregunta es ¿Cómo diseñar una

\footnotetext{
${ }^{1}$ Administrador Policial. Especialista en Pedagogía para la Educación Superior. Estudiante de la Maestría en Educación de la Universidad de la Sabana (Bogotá). Oficial de la Policía Nacional de Colombia en el grado de Mayor. Subdirector Escuela Carabineros Provincia de Vélez.

${ }^{2}$ Administrador Policial. Estudiante de la Maestría en Educación de la Universidad de la Sabana (Bogotá). Oficial de la Policía Nacional de Colombia en el grado de Capitán. Jefe de Planeación Escuela de Cadetes General Santander.

${ }^{3}$ Comunicadora social-periodista. Especialista en Servicio de Policía. Estudiante de la Maestría en Educación de la Universidad de la Sabana (Bogotá). Oficial de la Policía Nacional de Colombia en el grado de Capitán. Secretaria Privada de la Región de Policía Nro. 2 Sur Oriental.
} 
estrategia didáctica para la aplicación del Modelo Holístico de Liderazgo Policial (MHLP), desde la práctica docente, en el Técnico Profesional en Servicio de Policía de la Escuela de Carabineros Provincia de Vélez "Mayor General Manuel José López Gómez"?, y cuyo objetivo general se enmarca en el diseño de la misma. Este estudio toma como base la investigación cualitativa, bajo un enfoque hermenéutico, apoyados en el estudio de caso. En el proceso de análisis de resultados de entrevistas, análisis documental y observaciones, se ha encontrado que los docentes desarrollan en sus clases aspectos del liderazgo, pero no cuentan con el conocimiento del MHLP. Asimismo, el plan de estudios no tiene una asignatura propia de liderazgo, estas situaciones permiten generar nuevas herramientas didácticas y pedagógicas que permitan la interiorización del Modelo Holístico en los futuros policiales.

Palabras clave: práctica pedagógica, liderazgo, modelo holístico, estudiantes.

\title{
ADVANCES IN THE DESIGN OF A DIDACTIC STRATEGY FOR THE APPLICATION OF THE HOLISTIC MODEL OF POLICE LEADERSHIP - FROM THE TEACHING PRACTICE IN THE CARABINEROS SCHOOL OF VÉLEZ PROVINCE
}

\begin{abstract}
This article is an advancement of research related to the application of the holistic model of police (MHLP) of the police national leadership of Colombia (PONAL), in the school of Carabineros province of Vélez (ESVEL). In the case of the police training schools, even the MHLP processes have not developed. Therefore, before this need and as a contribution to the teachers of the school for Carabineros Velez province, is developing research work that precedes this article, the question is how to design a teaching strategy for the application from the holistic model of police leadership (MHLP), since the teaching practice in the professional technician in service of police from the school of Carabineros province of Velez "Major General Manuel José López Gómez"?, and whose overall objective is part of the design of the same. This study takes as a basis the qualitative research, under a hermeneutic approach, supported by case study. In the process of analysis of results of interviews, documentary analysis and observations, found that teachers develop aspects of leadership in their classes, but do not have the knowledge of the MHLP. Also the curriculum does not have an own leadership course, these situations can generate new didactic and pedagogical tools that permit the internalization of the holistic model in police futures.
\end{abstract}

Keywords: pedagogical practice, leadership, holistic model, students. 


\section{INTRODUCCIÓN}

En la Policía Nacional de Colombia, se ha venido generando ideas fuertes con relación al liderazgo en la Institución, que involucran no solo a los funcionarios uniformados, sino también a los administrativos en todos los niveles. Con el pasar de los días y los procesos en torno a la cultura institucional, se dio origen al Modelo Holístico de Liderazgo Policial (MHLP), el cual es fruto del trabajo investigativo del Señor Coronel Juan Carlos Nieto Aldana, Oficial de la Policía Nacional de Colombia.

El MHLP fue institucionalizado en junio de 2018. Desde entonces, se ha venido aplicando, poco a poco, en diferentes unidades policiales a lo largo y ancho del territorio colombiano. Dentro de esas dependencias, se incluye la Dirección Nacional de Escuelas (DINAE), la cual tiene a su cargo la orientación académica de 23 escuelas que existen en el país, y en 14 de ellas se ofrece el programa Técnico Profesional en Servicio de Policía (TPSP), el cual fue avalado con registro calificado por el Ministerio de Educación Nacional de Colombia.

Dentro de estas escuelas, se encuentra la Escuela de Carabineros Provincia de Vélez "Mayor General Manuel José López Gómez”, en la cual, como ya se ha mencionado, se está adelantando el TPSP, cuya resolución de aprobación de registro calificado fue otorgado mediante Resolución Nro. 11159 del 11/09/2012 del Ministerio de Educación Nacional.

En la actualidad y frente al MHLP, aún no se evidencian procesos de aplicación e implementación de este, en los jóvenes estudiantes que se encuentran en proceso de formación dentro de la escuela de policía en Vélez, por cuanto los docentes policiales que imparten las diferentes asignaturas del programa académico, no cuentan con el conocimiento apropiado y profundo del Modelo Holístico, cuya finalidad es mejorar el servicio de policía a través del liderazgo vivencial.

Como Institución, se están asumiendo los nuevos retos del país y, por consiguiente, implementando nuevas estrategias que permiten el acercamiento al ciudadano y responder los desafíos de convivencia ciudadana.

Por tal motivo, la Policía Nacional diseñó una estrategia a través del proceso de Modernización y Transformación Policial (MTI), la cual busca consolidar un cuerpo de policía más humano, integro, disciplinado, innovador y cercano al ciudadano, una realidad que se viene consolidando después de muchos esfuerzos en el MHLP, que busca fundamentalmente que cada uno de los integrantes de la PONAL, comprendan que es necesario liderar la vida propia, la familia, comunidad y, por ende, la Institución desde las diferentes labores que ellos mismos realizan al interior de ella. 
Es de reconocer que, la PONAL ha tenido un compromiso histórico con los ciudadanos, en la búsqueda de dar respuesta a sus necesidades en convivencia y seguridad ciudadana; por tal motivo, uno de los componentes en los cuales deben enfocarse las escuelas de policía, es en la transformación del servicio de policía o, dicho de otra forma, en la educación de sus hombres.

Para ello, se requiere no solo que los integrantes de la institución policial hoy tengan el conocimiento del MHLP, sino para quienes se encuentran en proceso de formación, que sea un eje fundamental en la implementación y continuidad del modelo, ya que ellos estarán en contacto permanente con su comunidad y, además, liderarán miles de procesos alrededor de ella. Para que este modelo dé frutos en sus estudiantes, se requiere de unos docentes comprometidos con el desarrollo académico y, sobre todo, con estrategias metodológicas apropiadas, que permitan una interiorización permanente con el modelo, en cada una de sus asignaturas, para poder contar con policías con alto grado de cultura institucional, formados en competencias de liderazgo óptimas para las necesidades del servicio de policía. (Policía Nacional, 2009)

Los autores del presente artículo, integrantes de la PONAL de Colombia y estudiantes de la Maestría en Educación de la Universidad de la Sabana, interesados en la forma de aplicar el MHLP en la Escuela de Carabineros Provincia de Vélez (ESVEL), se han venido preguntado ¿Cómo diseñar una estrategia didáctica para la aplicación del Modelo Holístico de Liderazgo Policial desde la práctica docente en el Técnico Profesional en Servicio de Policía de la Escuela de Carabineros Provincia de Vélez “Mayor General Manuel José López Gómez”? Por cuanto han descubierto que si bien los contenidos curriculares que se dan en el proceso de formación de los estudiantes del TPSP, presentan algunos elementos propios del liderazgo y, a su vez, los docentes cuentan con una formación apropiada en y para el liderazgo policial; no existe una asignatura relacionada con el liderazgo policial que desarrolle propiamente el modelo.

Sin embargo, en la consolidación de la formación policial, se han venido buscando estrategias apropiadas, originales y debidamente contextualizadas, que ayuden en la consecución de un uniformado que no solo contribuya con la seguridad, sino que sea un promotor de las realidades y vivencias, en donde la comunidad vea en ellos un generador de nuevas expectativas ante sus situaciones problema y un promotor de ayuda ante las dificultades. Por tal motivo, la Policía Nacional en el año 2018, a partir del MHLP, determinó un nuevo concepto de ciencia de policía que permite integrar los ámbitos personal, institucional y comunitario del uniformado con atributos claves, como el honor policial, la visión, la innovación, la credibilidad, la confianza, la vocación de servicio, la adaptabilidad y la efectividad, elementos esenciales para optimizar el servicio de policía y movilizar la cultura institucional frente a los entornos sociales dinámicos y complejos de 
la sociedad colombiana. Estos atributos tienen una aplicación que recae sobre los ámbitos donde el uniformado desempeña su función a diario. En lo personal: "Yo lidero mi vida". En lo institucional: "Yo lidero mi Institución". Y en lo comunitario: "Yo lidero mi comunidad" (Policía Nacional, 2018, párr. 2).

Si bien el MHLP comenzó su implementación hace poco tiempo, la PONAL ha venido desarrollando su proceso de formación de los estudiantes del TPSP, a partir de tres pilares fundamentales, relacionados con la potenciación del conocimiento, el enfoque humanista y la formación integral, mediante los momentos educativos de formación, actualización, entrenamiento y capacitación, con los que se pretende avanzar hacia un proyecto de profesionalización, profundización y conocimiento permanente de la ciencia policial, articulado a un modelo pedagógico institucional con enfoque educativo por competencias. En este sentido, el Proyecto Educativo Institucional "plantea un enfoque que responde a las necesidades de la institución y del país, para aportar mejores hombres y mujeres al servicio policial” (Policía Nacional, s.f., p. 19).

Sin embargo, a pesar de que el modelo educativo no ha logrado alcanzar un grado máximo, en donde el liderazgo sea un motivo fundamental en la consolidación de las escuelas y un motor en el desarrollo pedagógico del programa, ello implica la necesidad de reflexionar sobre la educación policial a partir del perfil del profesional de policía que se requiere, tanto para enfrentar los desafíos que presentan las dinámicas actuales de convivencia y seguridad ciudadana, como para asumir los procesos de cambio que se dan en el proceso de renovación policial (Bulla \& Guarín, 2015; Tolosa, 2016).

La formación en liderazgo de los futuros policías de Colombia, es esencial para la prestación de su servicio, teniendo en cuenta que son ellos quienes estarán atendiendo de primera mano las necesidades de la comunidad en materia de convivencia; por tal motivo, es importante que los docentes formadores y el currículo, estén mucho más permeados por los fundamentos del MHLP.

Los docentes formadores requieren de un perfil apropiado para el servicio de formación policial, el cual, no solo debe poseer sus respectivas titulaciones, sino que se exige experiencia en docencia universitaria, conocimientos específicos en TIC (deber ser ciudadano o maestro digital), dominio de una lengua foránea, experiencia profesional y docente (en IES), experiencia investigativa, con evidencia de vinculación a grupos de Colciencias, producción intelectual, en donde demuestre el aspirante que ha participado en la construcción de documentos de orden institucional o de programas (Policia Nacional de Colombia, 2017) 
Adicionalmente, se requiere de estrategias propias para la aplicación del modelo, entendidas estas como los procedimientos o recursos utilizados por los docentes para lograr aprendizajes significativos en los alumnos. "Cabe hacer mención que, el empleo de diversas estrategias de enseñanza permite a los docentes lograr un proceso de aprendizaje activo, participativo, de cooperación y vivencial. Las vivencias reiteradas de trabajo en equipo cooperativo hacen posible el aprendizaje de valores y afectos, que, de otro modo, es imposible de lograr" (Nolasco, s.f., párr. 4).

Para el presente, los docentes aplican diferentes estrategias de aprendizaje para el desarrollo de sus clases, que coinciden con los aspectos mencionados anteriormente, algunas de estas estrategias son: análisis de casos, talleres, práctica, lecturas, proyectos, guías de aprendizaje, seminarios, ensayos, entre otros, que buscan que el aprendizaje de los jóvenes sea vivencial, actualizado y acorde con las necesidades del entorno.

Para este trabajo, que a primer semestre de 2019 aún se encuentra en proceso de investigación, metodológicamente se partió de un enfoque cualitativo y se fundamenta en los datos la sistematización de datos no estadísticos, bajo un enfoque hermenéutico.

Se ha buscado a través de ella, generar espacios de indagación de los problemas asociados con el liderazgo y su incidencia en las prácticas docentes que se han venido orientando en la ESVEL, en este sentido, el analizar las perspectivas de los informantes puede conducir a comprender los diversos significados que se dan al MHLP.

Para tal fin, es importante reconocer en la comunidad policial la importancia del liderazgo público y como se debe proyectar en los nuevos policías, lo que requiere de una perspectiva institucional objetiva, capaz de comprender la complejidad humana y desde un concepto antropológico la condición del ser humano y su relación con su hábitat, territorio y sociedad, partiendo de la base misma que antes de ser policía es un ser humano dotado con una autoridad, para ser el garante de los deberes y derechos constitucionales que todos los ciudadanos merecen y deben respetar.

La DINAE, con el fin de profesionalizar a los jóvenes que ingresaban a la Institución y con la intención de buenas y mejores prácticas de liderazgo por parte de los integrantes de la Policía Nacional, buscando ante todo efectividad, consolidación de un cuerpo de policía más humano, integro, disciplinado, innovador, cercano al ciudadano y comprometido con los nuevos retos de seguridad y convivencia del país; gestionó ante el Ministerio de Educación Nacional de Colombia, 
la creación e implementación del programa Técnico Profesional en Servicio de Policía (TPSP).

\section{PROGRAMA TÉCNICO DE SERVICIO DE POLICÍA}

El TPSP como programa de formación para la educación superior, fue creado por la PONAL en atención a las necesidades de la institución policial, en cuanto a la profesionalización de sus futuros funcionarios en el nivel operativo. Este programa se desarrolla en metodología presencial y va dirigido a jóvenes entre 18 a 25 años en diferentes contextos culturales. En la actualidad, se desarrolla en las 14 Escuelas de formación de la PONAL.

Como programa de formación universitaria, se alinea con las necesidades del país y del entorno, permitiendo así generar competencias en los hombres y mujeres policías, para el mejoramiento de la convivencia ciudadana.

El programa está orientado desde una perspectiva interdisciplinar, de manera que se logre la formación de un policía integral con conocimientos sólidos en su formación específica, los valores y alto sentido de la ética, para que pueda dar respuesta a los planteamientos de la misión y los objetivos, para garantizar la convivencia pacífica y seguridad ciudadana.

Por ello, "resulta importante para la educación y el servicio de policia mejorar las prácticas pedagógicas en la Policía Nacional, así como contar con una doctrina educativa para el docente policial con énfasis en la instrucción” (Londoño, Patiño, Rodríguez \& Gil, 2018, p. 214), situación que está definida en el modelo pedagógico institucional.

Este modelo manifiesta que, dentro de las disciplinas que aportan a la formación del estudiante, se encuentran: la sociología, antropología, el derecho, mediante aproximaciones psicológicas, sociológicas y políticas de las organizaciones sobre las cuales versa su estudio (Policía Nacional, s.f.).

Además, el programa en el modelo pedagógico policial cuenta con un enfoque educativo por competencias, entendidas como las habilidades, destrezas, actitudes, aptitudes, valores que debe tener un profesional para ejercer en los diferentes contextos.

El programa asume como propias once competencias transversales, que pueden verse en la siguiente figura (ver figura 1). 


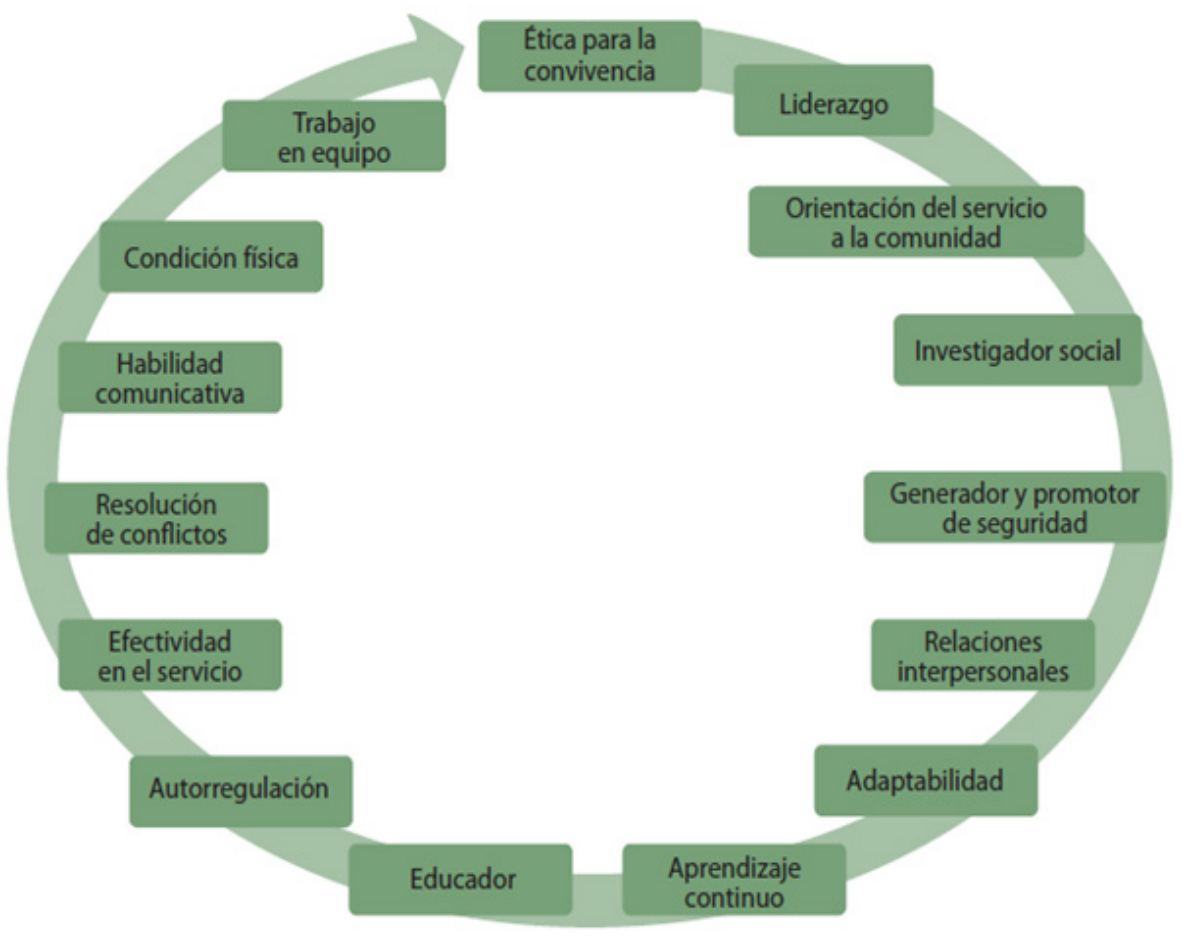

Figura 1. Competencias Transversales de la Educación Policial.

Fuente: Quintero, García, \& Orozco, (2010) p. 57

Dicho modelo de formación busca fortalecer las competencias con relación a la formación policial, y comprende los siguientes elementos: contexto, actores educativos fundamentales: estudiantes y docentes, programas, aprendizaje basado en competencias.

Este enfoque educativo busca que el funcionario desarrollo las por competencias en el ser, el saber, el saber hacer y el saber convivir, requeridos para formar un policía competente e integral, de acuerdo con las necesidades sociales e institucionales (Policía Nacional, 2006). El enfoque por competencias es útil en el campo educativo, porque al estudiante no se le exige solamente que repita una información, sino que demuestre que está en capacidad de utilizar esa información en el momento oportuno, para resolver un problema o realizar una tarea (Policía Nacional, 2012).

El papel del profesional de policía se ha transformado. Actualmente, un policía competente es aquel que se desempeña adecuadamente en contextos laborales propios de la profesión, resolviendo en forma responsable, autónoma y flexible, las situaciones que se le presentan en el ejercicio de sus funciones; pero que, además, transforma positivamente su organización, lugar de trabajo, y, en general, su entorno (Policía Nacional, 2012). 


\section{MODELO HOLÍSTICO DE LIDERAZGO POLICIAL (MHLP)}

El MHLP busca articularse e impulsar la misión constitucional, permitiendo la adaptación a nuevos entornos dinámicos, en un mundo en constante evolución, y en donde se requieren uniformados líderes con características y competencias necesarias que permitan direccionar y asegurar la proyección de la institución.

La labor policial está claramente enmarcada en escenarios que requieren ser abordados a través de un modelo de liderazgo institucional propio. El liderazgo se debe considerar un factor determinante para orientar y dirigir una institución, logrando transmitir seguridad al equipo de trabajo, pues mientras más seguros se sientan en una organización más comprometidos estarán con sus labores cotidianas. "La Policía Nacional fomenta la profesionalización del hombre y mujer policía, formando líderes competentes interesados en investigar e innovar, para la mejora permanente del servicio policial" (Policía Nacional, 2015, p. 18).

Las necesidades de formación, actualización y capacitación, que surgen del día a día en el desempeño policial, son el insumo constante para retroalimentar los procesos educativos, y el factor básico para reestructurar y crear programas. Por lo ello, el MHLP busca preparar a la institución para los retos del futuro, respondiendo de manera oportuna y efectiva a las exigencias de la dinámica social; por lo tanto, se viene implementando un proceso de modernización de la Policía, lo suficiente flexible para asumir los cambios permanentes del entorno, y así adoptar y generar avances para su desarrollo.

A través del programa Técnico Profesional en Servicio de Policía y de sus competencias iniciales cuando son seleccionados, los estudiantes inician un proceso de formación en competencias transversales, resaltando entre estas el liderazgo, el cual busca formar integralmente al futuro profesional de Policía partiendo de sus habilidades destrezas y actitudes, que permitan apropiarse de los conocimientos e integrarlos a su aprendizaje.

\section{ENTORNOS DEL MLHP}

El modelo ha denominado entornos, a los medios o atmósferas en los que, por su naturaleza, el funcionario debe desempeñar sus labores y ejercer su liderazgo frente a los objetivos y responsabilidades propias. Nieto, Nieto y Moreno (2018) expresan que estos entornos son:

Operativo: se consideran como entornos operativos, las circunstancias para contrarrestar el accionar de la delincuencia común y organizada, la prevención y conservación del orden público y la convivencia pacífica, las misiones de vigilancia e inteligencia, la policía judicial, el servicio 
aéreo, los grupos de reacción y operaciones especiales, las labores primarias del servicio policial, la lucha contra el secuestro y la extorsión, la policía comunitaria, la Policía Fiscal y Aduanera, la policía de tránsito y carreteras y otras comisiones oficiales de carácter internacional. (p. 97).

Desde esta perspectiva, se debe privilegiar el papel del docente como transcendental para lograr un liderazgo de influencia positiva, y con el convencimiento de que al formar un mejor ser humano, este profesional de policía estará en la capacidad de generar las condiciones de convivencia que la sociedad requiere, además de admitir personas abiertas a la innovación con una gran creatividad, capaces de analizar y enfrentar situaciones complejas ajustadas a la legalidad, de desarrollar nuevas habilidades, procesos de comunicación, pero, sobre todo, un gran liderazgo para las exigencias de la función policial. Para efectos del presente modelo, se denominaron entornos, a los medios o atmósferas en los que, por su naturaleza, el funcionario debe desempeñar sus labores y ejercer su liderazgo frente a los objetivos y responsabilidades propias (Policía Nacional, 2017).

Administrativo: Los entornos administrativos se refieren al conjunto de circunstancias en las cuales se desarrollan tareas relacionadas con la administración de recursos, talento humano, servicio y apoyo, servicios médicos y hospitalarios, cumplimiento de las metas estratégicas de la institución y apoyo a organismos de la administración pública e interinstitucionales. (p. 97).

Es decir, cómo se realiza la docencia en el programa, cuántos docentes se encuentran, cuáles son las estrategias actuales que desarrollan para orientar sus clases, cuál es el perfil del docente, no en lo disciplinar sino entorno a lo específico del liderazgo.

Docente: los entornos docentes, son aquellas circunstancias y condiciones laborales, en las cuales se desarrollan actividades de formación, capacitación y especialización en las diferentes escuelas de formación policial. Culturas de liderazgo relevantes en la Policía Nacional. Para efectos del presente modelo se entiende por culturas de liderazgo en la Policía Nacional de Colombia, los comportamientos o hábitos más comunes, producto de los estilos de liderazgo predominantes en la institución. (p. 97).

"Para que una organización sea exitosa en el entorno actual, necesita mantener su nivel de urgencia de mediano a alto" (Kotter, 2011, p. 45). Esto quiere decir que el liderazgo, se debe considerar un factor determinante para orientar y dirigir una institución, logrando transmitir seguridad al equipo de trabajo, pues mientras más seguros se sientan en una organización, más comprometidos estarán con sus labores cotidianas. 
Las necesidades de formación, actualización y capacitación, que surgen del día a día en el desempeño policial, son el insumo constante para retroalimentar los procesos educativos y el factor básico para reestructurar y crear programas. Por lo ello, el MHLP busca preparar a la institución respondiendo de manera oportuna y efectiva a las exigencias de la dinámica social; por lo tanto, se viene implementando un proceso de modernización de la Policía, lo suficiente flexible para asumir los cambios permanentes del entorno, y así adoptar y generar avances para su desarrollo. Es así que, el liderazgo llega a la institución como parte de una herramienta didáctica vital en los procesos de formación académica de los policías en formación.

Frente a esto, es importante destacar la efectividad que se ha venido dando en los policías en formación.

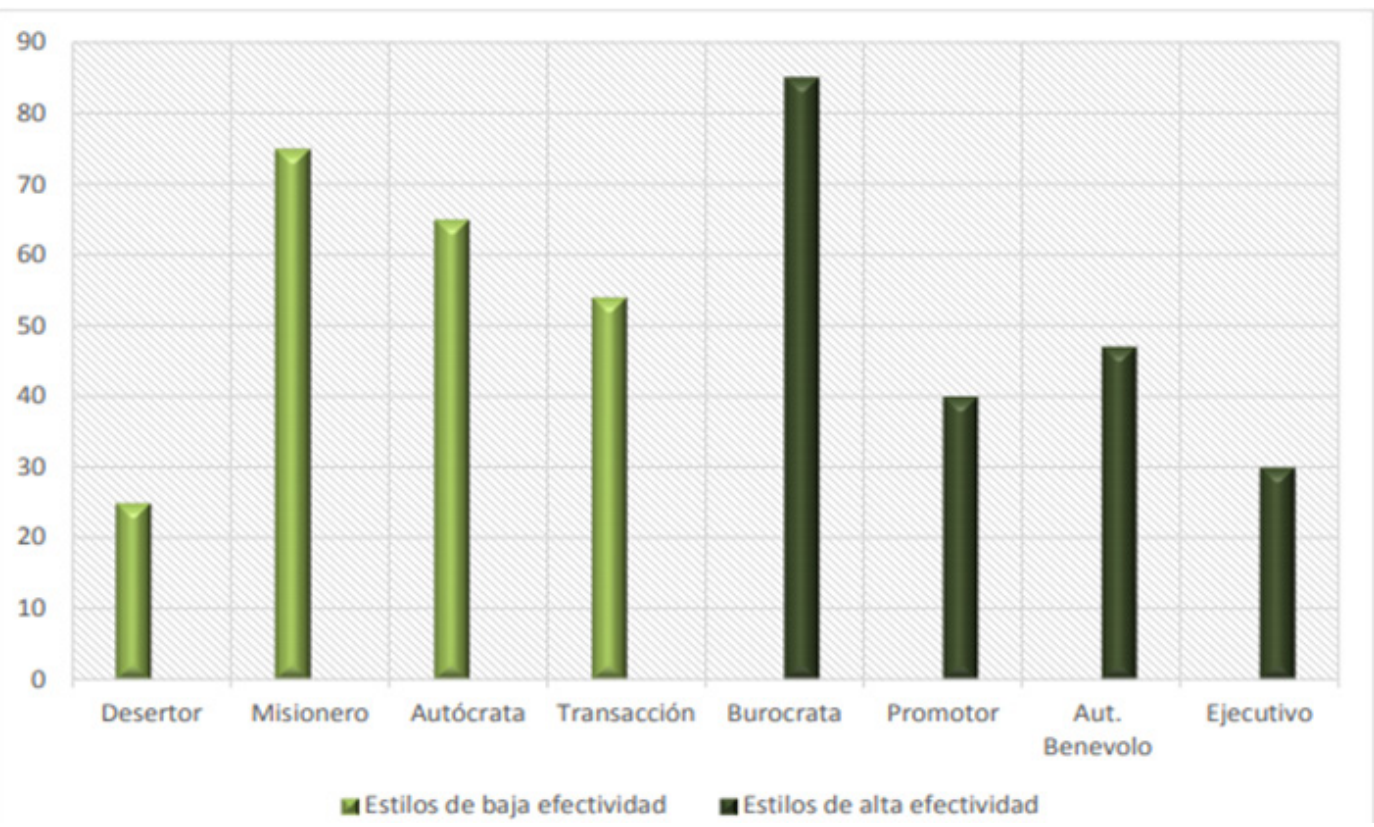

Figura 4. Resultados obtenidos de Liderazgo identificados en la institución.

Fuente: Nieto et al., 2017 p. 101.

De acuerdo con la gráfica, el estilo de liderazgo de alta efectividad llamado Burócrata y los dos estilos de baja efectividad llamados Misionero y Autócrata, son los que más se destacan. Esto significa que la institución se mueve con un liderazgo ordenado y eficiente la mayor parte del tiempo; y, cuando está sometido a presión, afloran estilos de liderazgo de baja efectividad en virtud de los cuales se suele ser tolerante con el bajo nivel de desempeño (alto nivel de cumplimiento) y alternativamente extremamente intolerante, lo que da un cuadro de inestabilidad que tiende a irritar el clima de trabajo. 


\section{Tabla 1}

Concepto OT y OR

\begin{tabular}{|c|l|}
\hline ORIENTACIÓN A LA TAREA (OT) & $\begin{array}{l}\text { Es el grado en que un gerente dirige sus } \\
\text { propios esfuerzos y los de sus subordinados, } \\
\text { se caracteriza por iniciar, organizar, planear, } \\
\text { controlar y dirigir. }\end{array}$ \\
\hline ORIENTACIÓN A LAS RELACIONES (OR) & $\begin{array}{l}\text { Es el grado en que un gerente tiene relaciones } \\
\text { personales en su tarea; se caracteriza por } \\
\text { escuchar, confiar, preocuparse por los } \\
\text { sentimientos de otros y estimular. }\end{array}$ \\
\hline
\end{tabular}

Fuente: adaptado de Reddin (1983).

Cada estilo de liderazgo genera diferentes niveles de efectividad dependiendo de la situación, abarcando todos los ámbitos del líder policial: personal, institucional y de servicio a la comunidad. Por lo tanto, esta teoría trabaja bajo dos elementos primordiales como: orientación a la tarea (OT) y orientación a las relaciones personales (OR), permitiendo que las personas independientemente de los diferentes estilos de liderazgo, se comporten efectivamente dependiendo de la situación a la que se enfrenten.

\section{METODOLOGÍA DEL PROCESO}

Para la presente investigación, se ha asumido el enfoque cualitativo, que, en palabras de Martínez (2006), hace referencia al "estudio de un todo integrado que forma o constituye una unidad de análisis y que hace que algo sea lo que es: una persona, una entidad étnica, social, empresarial, un producto determinado" (p. 126). Adicionalmente a ello, los autores han asumido como posición conceptual, la expresada por Marín (2012):

Seguir un proceso inductivo deductivo. En donde los investigadores desarrollan conceptos, intelecciones y compresiones de la realidad, para que a partir de premisas generales las llevan a los casos particulares para iluminar la realidad y transfórmala en cuanto sea posible. De la misma forma se considera más flexible que la investigación cuantitativa pero no menos rigurosa. (pp. 124$125)$.

La implementación MHLP en la Escuela de Carabineros Provincia de Vélez, evidencia que en ella, se está inmerso en un problema mucho mayor relacionado con el liderazgo policial y los elementos necesarios para que se desarrollen los diferentes componentes en los procesos académicos que se llevan al interior de esta; lo que permite corroborar lo manifestado por Mendoza (2006), cuando afirma, con respecto a la orientación metodológica, que: “ [...] no suele partir del planteamiento de un problema especifico, sino de un área problemática más amplia en la 
Avances del diseño de una estrategia didáctica para la aplicación del Modelo Holístico de Liderazgo Policial MHLP, desde la práctica docente en la Escuela de Carabineros provincia de Vélez Jesús E. Villamizar S. - Sandra P. Riveros G. - Diomayra L. Vacca G. (11:27)

cual puede haber muchos problemas entrelazados que no se vislumbrarán hasta que no haya sido suficientemente avanzada la investigación." (p. 131).

El proceso de categorización implicó la consolidación de dos metacategorías, que son: liderazgo policial, cuyas categorías fueron perfil policial y apropiación del modelo; y práctica docente. Dentro de la misma, se consolidaron las categorías contenidos curriculares y práctica didáctica y pedagógica.

En este punto, el método de esta investigación de es de tipo hermenéutico. El cual se pregunta sobre el sentido y el significado que tiene para sus actores. Según Ebeling (citado en Marín, 2012), "el término hermenéutica designa la ciencia o el arte de interpretación con los siguientes significados: 1. expresar, en el sentido de afirmar, hablar; 2. explicar, que significa interpretar, aclarar; y 3. traducir, que quiere decir trasladar" (p. 127).

Por su parte, para Latorre, Rincón y Arnal (2005), la hermenéutica busca comprender e interpretar la realidad desde la visión de un grupo de personas en sus diversos contextos, para este estudio en específico la realidad policial en el proceso de formación del Técnico Profesional en Servicio de Policía orientado a los futuros patrulleros de la Policía Nacional. En este sentido y siguiendo a Taylor \& Bogdán (1996), es importante aclarar que este método es un proceso inductivo, que tiende a ser holístico, en donde los investigadores se ven afectados por los eventos propios del estudio, se busca comprender a las personas dentro del marco de referencia (contexto de interacciones sociales y cotidianas), y finalmente el investigador aporta en el proceso (creencias, perspectivas y predisposiciones).

Frente a esto, es importante hacer énfasis en que no existe una realidad única en la formación del policial referente al programa académico del técnico profesional en servicio de policía, y por ende de las interacciones sociales de los sujetos, que, para este estudio en específico, son las personas que se desempeñan como docentes policiales en la Escuela de Carabineros Provincia de Vélez “Mayor General Manuel José López Gómez”.

Para lo cual, cabe resaltar que la realidad es distinta en cada una de las escuelas de formación policial, en donde el plan de estudios es igual para las 13 escuelas y 1 centro de instrucción a nivel nacional, en donde su régimen de disciplina y proceso de formación policial es el mismo, orientado bajo el Técnico Profesional en Servicio de Policía, lo que varía es el lugar y contexto en donde se encuentra la escuela policial. En este sentido, es importante comprender que todo conocimiento adquirido contiene un significado cuando se da en un contexto o situación determinada. 
Finalmente, en la hermenéutica interpretativa, puede decir que se ponen en juego las dos narrativas, que a veces se mezclan, se confunden o se solapan. Estas son las narraciones que hacen los sujetos sociales acerca de sus prácticas, por lo que el proceso de formación policial se orienta al estudiante para la acertada toma de decisiones, frente a las acciones que desarrollará en su ejercicio profesional como futuro profesional de policía.

Es decir, se utiliza como ejemplo el estudio de caso, la casuística, entre otras estrategias educativas, que le permitirán al estudiante fortalecer su orientación educativa, a través de un ejercicio académico por parte de los docentes, mediante su acto educativo dirigido al personal estudiantil.

El estudio de caso desde la perspectiva Eisenhardt (citado en Martínez, 2006), concibió el estudio de caso contemporáneo como: "una estrategia de investigación dirigida a comprender las dinámicas presentes en contextos singulares", la cual podría tratarse del estudio de un único caso o de varios casos, combinando distintos métodos para la recogida de evidencia cualitativa y/o cuantitativa con el fin de describir, verificar o generar teoría, lo que puede considerarlo como un campo innovador para el desarrollo del conocimiento científico, sobre todo en el terreno de la investigación educativa y de comunicación, donde su uso es escaso por el desconocimiento de sus ventajas, por lo que este artículo es un espacio de diálogo para los interesados en el tema (p. 37).

El caso debe ser estudiado holísticamente, y no restringirse a ciertas áreas o algunos cuantos lugares o personas, el cual aporta descripciones densas y ricas con el propósito de interpretar y teorizar sobre el caso. El modelo de análisis es inductivo, para desarrollar categorías conceptuales que ilustren, ratifiquen o desafíen presupuestos teóricos difundidos antes de la obtención de la información.

Finalmente, las técnicas a desarrollar tienen que ver con los procedimientos utilizados para la recolección de los datos, es decir, el cómo. Estas fueron la revisión documental, observación y entrevista.

En la revisión documental, se logró determinar que no existen muchos documentos institucionales que hablen del MHLP. Adicionalmente a ello, el plan de estudios evidencia la no existencia de una asignatura propia del modelo, sin embargo, en las guías de observación aplicadas a los docentes policiales, se logra comprobar la existencia de elementos propios del liderazgo policial, que si bien no están debidamente definidos como lo establece el modelo, sí permiten ver que ya hay unos primeros avances de integración del mismo en el TPSP. 
La guía de observación utilizada en el presente trabajo como instrumento de aplicación, se aplicó a siete docentes de la Escuela Carabineros Provincia de Vélez, realizando una observación en sus clases (cuatro asignaturas del primer período académico y tres asignaturas del segundo período académico), incluyendo los siguientes campos de formación: humanista e investigativa, jurídica, policial y electiva.

En esta guía de observación, se encontró que en cada una de las siete clases observadas, puede comprobarse que los docentes utilizan una serie de estrategias debidamente contextualizadas con relación a las situaciones que viven los policías en la cotidianidad de su labor, priorizando dentro del proceso de enseñanza el desarrollo de estudios de caso a través de los cuales se ubica al estudiante en situaciones reales, para determinar, a partir del saber adquirido, qué decisiones debe tomar en el saber hacer, generando debate y retroalimentación entre los aciertos y desaciertos identificados en la atención de los diferentes casos de policía que hacen parte del quehacer diario del hombre y mujer policía, permitiendo generar una concepción de los distintos escenarios que deberán atender en el marco de su desempeño policial.

Frente a las dos entrevistas realizadas al señor Coronel de la Policía Nacional Juan Carlos Nieto Aldana y a la Doctora Laura Nuñez Flechert, se evidencia que ambos participantes insisten en la importancia del MHLP, en la aplicación paulatina del mismo al interior de la Institución y que una de las necesidades fundamentales, es llegar a las jóvenes que inician la carrera policial a través de las Escuelas de formación. Por ello, surge la oportunidad de comenzar con estos procesos investigativos al interior de estas, y brindar las mejores estrategias a los docentes para la aplicación del modelo.

\section{CONSIDERACIONES FINALES}

El proceso de avance institucional (puede decirse que la PONAL) ha buscado integrarse de manera eficaz en diferentes procesos educativos, lo que le permitió desde hace varios años la creación de una Institución Universitaria, que le ha dado la opción de graduar como técnicos en servicio de policía, a los uniformados que hoy se encuentran en los diferentes distritos policiales del país.

Este proceso en búsqueda de calidad, también ha generado que se cuente a 2019 con programas acreditados en alta calidad para el desarrollo integral y competente de sus profesionales de policía, bajo los estándares de supervisión permanente y mejora continua. Teniendo en cuenta que la formación de los policiales no cuenta con una asignatura propia relacionada con el liderazgo policial, también se hace necesario verificar si los contenidos curriculares presentan elementos 
propios del liderazgo y que se enseñan a los estudiantes de las escuelas. De la misma forma, si los docentes cuentan con una formación apropiada en y para el liderazgo policial.

El MHLP implica la utilización de todos los estilos de liderazgo existentes de acuerdo con el nivel de responsabilidad del funcionario, en los diferentes campos de acción en los cuales se desempeñe, como son, dirección, asesoría, administración y gestión, contribución individual superior, y contribución individual básica; cada una de ellas contribuye a la construcción de una Institución debidamente consolidada desde el logro del liderazgo. Teniendo en cuenta que uno de los procesos donde se debe hacer énfasis para alcanzar altos niveles de convivencia en una sociedad, es la educación, base fundamental, donde hay conexión directa para fortalecer el aprendizaje, la pedagogía social y la socialización aplicada a los nuevos contextos de paz.

Los ciudadanos que desean ser policiales, son formados en el TPSP, que se ofrece en las diferentes escuelas del país, programa de formación que se encuentra avalado por el Ministerio de Educación Nacional.

Se hace necesario desde esta perspectiva, privilegiar el papel del docente como transcendental para lograr un liderazgo de influencia positiva, y con el convencimiento de que al formar un mejor ser humano, este profesional de policía estará en la capacidad de generar las condiciones de convivencia que la sociedad requiere, además de admitir personas abiertas a la innovación con una gran creatividad, capaces de analizar y enfrentar situaciones.

\section{BIBLIOGRAFÍA}

Latorre, A., Rincón, D., \& Arnal, J. (2005). Bases metodológicas de la investigación educativa. Barcelona: Hurtado.

Londoño, G., Patiño, J., Rodríguez, D., \& Gil, B. (2018). Método de Instrucción Policial. Logos Ciencia \& Tecnología, 10(4), 207-218. doi: http://dx.doi.org/10.22335/rlct.v10i4.753.

Marín, D. (2012). La investigación en educación y pedagogía. Bogotá D.C: USTA.

Martínez, M. (2006). La Investigación Cualitativa (Síntesis Conceptual). Investigación en Psicología, 123-146.

Nieto, J. (2018). Modelo Holístico de Liderazgo Policial. Bogotá, D.C.: Universidad EAN.

Nieto, J., Nieto, J., \& Moreno, J. (2018). Modelo holístico de liderazgo policial. Revista Logos Ciencia \& Tecnología, 10, 90-110.

Nolasco, M. (s.f.). Estrategias de enseñanza en educación. Recuperado de https://www.uaeh. 
edu.mx/scige/boletin/prepa4/n4/e8.html

Policía Nacional de Colombia (2006). Resolución 01751 de 2006 por la cual se implementan las competencias genéricas en la Policía Nacional. Bogotá D.C.: Policía Nacional de Colombia.

Policía Nacional de Colombia. (2009). Doctrina Educativa Para El Docente Policial. Editorial Líneas Creativas, Publicaciones Ltda. S.A. Recuperado de http://www.policia.edu.co/ documentos/2017/doctrina/Doctrina\%20educativa\%20para\%20el\%20docente\%20policial\%20 2009.pdf

Policía Nacional de Colombia. (2015). Plan Estratégico Institucional Comunidades Seguras y en Paz Visión 2030. Bogotá: Policía Nacional.

Policía Nacional de Colombia. (2017). Convocatoria docente contrato 002. Recuperado de https://www.policia.gov.co/sites/default/files/convocatoria-002-docentes-contrato-laborarescuela-suboficiales-mandos-ejecutivos.pdf

Policía Nacional de Colombia. (s.f.). Oficina de comunicaciones Estratégicas COEST - Misión Recuperado de https://www.policia.gov.co/unidad/oficina-de-comunicaciones-estratégicas-0

Policía Nacional. (2018). Conozca el liderazgo holístico policial. Recuperado de https:// www.policia.gov.co/noticia/conozca-liderazgo-holistico-policial

Policía Nacional. (2017). Educación base de la transformación institucional. PNC, pág. 20.

Policía Nacional. (s.f.). Lineamientos generales de Política para la Policía Nacional de Colombia. Bogotá: Policía Nacional.

Quintero, J. García, D; Orozco, O. (2010). Proyecto educativo institucional. Policía Nacional de Colombia. Potenciación del conocimiento y formación Policial. Editorial Líneas Creativas, Publicaciones Ltda. S. A.

Taylor, S., \& Bogdán, R, B. (1996). Introducción a métodos cualitativos de investigación. Madrid, España: Paidós. 